\title{
Implementing AODV Routing Protocol in VANET using SDN
}

\author{
Khandaker Mohammad \\ Mohi Uddin \\ Lecturer, Dept. of CSE, \\ Dhaka International University, \\ Dhaka, Bangladesh
}

\author{
Nayeema Islam \\ Assistant Professor, Dept. of \\ CSE, \\ Jagannath University, Dhaka, \\ Bangladesh
}

\author{
Jahanara Akhtar \\ Associate Professor, Dept. of \\ CSE, \\ Dhaka International University, \\ Dhaka, Bangladesh
}

\begin{abstract}
Software-defined network (SDN) is a new network architecture that is more manageable, adaptable, dynamic and programmable. Though the control plane is separated from the forwarding plane and it is directly programmable these make the network to become fast and easy to manage. SDN technology increases network capability and shortens the network configuration and resource management for its programmable features. For forwarding functions and centralized control, SDN supports Open-Flow technology. There are two forms of routing protocols used in Vehicular ad hoc networks (VANET) such as Proactive routing protocol and Reactive routing protocol. The Reactive routing protocol shows the best performance than others when two communicating devices are linked with each other. In this work, Ad hoc On Demand Distance Vector (AODV) is implemented as a reactive routing protocol in SDN and analyzes the performance based on using the most essential quality of service (QoS) metrics. Moreover, it is found that for VANET in SDN architecture, the highest packet delay is inferior to other traditional network architectures and the packet transfer rate is also high.
\end{abstract}

\section{General Terms}

AODV routing protocol, SDN architecture, Traditional network, Open-Flow technology

\section{Keywords}

SDN, Programmable, Reactive, Open-Flow, VANET, AODV

\section{INTRODUCTION}

SDN has been observed as a crucial permissive technology for the upcoming network [1]. Based on the Open-Flow protocol, the control plane and data plane are separated in SDN that promises to gain a significant improvement of the network performance. Packet forwarding and controlling perform in the same device in a traditional network system that generates the transmission curtail and is unable to adapt to the spreading threat of network topology. To accomplish emerging network necessities, a more bendable architecture is invented by SDN using the Open-Flow controller upon traditional network infrastructure. A software or hardware-based centralized controller manages the data controlling actions, and a hardware core device performs data forwarding tasks [2]. Though the control plane is directly programmable, SDN is suitable in the field of research. QoS features are contained by data plane functionality [3] which are used for measuring the performance of network topology. The preeminent aspiration of SDN is to design and form a network with the highest augmentation of QoS parameters.

VANET is an emerging class from MANET [4] where communication nodes are vehicles and these vehicles are equipped with calculators, sensors, and wireless communication technologies. At present, VANET is an active research area, which takes the attention of governments, industries, academics, and research communities [5]. Two types of routing protocols are used by VANET and reactive routing protocol is one of them. Though reactive routing protocol used dynamic table it is known as an on-demand routing protocol. AODV [6] and DSR (Dynamic Source Routing) are the reactive routing protocol. After studying about VANET routing protocol it can be said that the reactive routing protocol provides better performance than others. In this work, a reactive routing protocol AODV has been implemented in SDN and analyzed the performance based on some QoS parameters.

\section{RELATED WORK}

Currently, significant research works have undertaken to analyze the performance of different VANET protocols using different parameters and conditions. Nowadays, SDN is a new field for research.

Peng L. et al. [7] compared the performance of Proactive routing protocol in a more realistic city scenario basis of average packet ratio and average end delay. They used NS2.32 for their simulation. The output of their thesis is that AODV is more suitable for VANET and DSR.

Fang X. et al. [8] compared AODV and DSR in the VANET city scenario on basis of throughput and end-to-end delay with varying density. They observed that AODV is better than DSR when the number of vehicles are increased. They also found that AODV provides sufficient throughput with lower delay.

In [9] Shukla R.S. et al. Observed the performance of reactive routing protocol basics of throughput and end-to-end delay in VANET using a realistic mobility model. They used NS2 to measure the performance. Their result is that AODV performs then other routing protocols in VANET.

In [10] V. RAJESHKUMAR, P. SIVAKUMAR studied and analyzed the performance of AODV, DSDV and DSR Routing Protocols in MANET. They showed that AODV performance is the best considering its ability to maintain connection by periodic exchange of data. AODV and DSR showed a better performance than DSDV when throughput is concerned and the network has a large number of nodes. Habib M, Rutuja K [11] studied and implemented the DSDV routing protocol in SDN and analyzed the performance. They found that DSDV performs better in SDN than other traditional network architectures. Theoretically, SDN gives the best performance from other network architectures because the data plane and control plane are separated in 
SDN. Besides, AODV is better than other routing protocols like DSR and DSDV. In this work, AODV routing protocol is implemented in SDN and analyze the performance based on using (QoS) metrics.

\section{SDN and VNET}

In the conventional infrastructure, maintenance is very expensive for the variety and the complexity [12] of network elements. Frequent network failures make the underlying infrastructure-less reliable. Overall network implementation, configuration, and troubleshooting require a high-level technically skilled network in conventional infrastructure. Networking elements (e.g., routers, switches, and access points) take routing and forwarding decisions, these decisions are separated from the data plane in SDN. SDN supports programmability, centralized control, network flexibility, efficient configuration, and enhanced management.

The centralized control operation of SDN dictates the network policies. In SDN, Many controller platforms are used which are open source. Some controller platforms are as follows: Floodlight [13], OpenDayLight, and Beacon. There are different layers in-network (i.e., application, control, and data plane) and these layers manage the network. Customers get the resources through the application layer, Control plane helps to change the network policies and logical entities and the data plane help to set up physical network elements.

VANETs technology is the newest wireless network for vehicles. It merges the facilities of wireless networks. VANET works creating a robust Ad-Hoc network between roadside units and vehicles. It is a part of MANET and can take part to create a network between moving nodes by using some ad-hoc networking tools such as IEEE $802.11 \mathrm{bg}$ (Wifi), IEEE 802.10 (WiMAX), Bluetooth.

The aspiration of VANET is to grant safety-related information, supervise the traffic and involve the speed of communication. On the road, two moving vehicles can communicate with each other using VANET. Due to highspeed nodes, it is quite harder to maintain handoff.

\section{ROUTING PROTOCOL}

Routing is a process to discover an optimal path from source to destination. Diverse routing algorithms are used to find out the optimal path. VANET is a part of MANET, where MANET is implemented in VANET to handle mobility scenarios and improve the network performances. There are two major challenges to find out the optimal path in VANET [14]. The first one is, High mobility of nodes and the second one rapidly changes in topology.

Routing protocols in VANET are classified in 3 ways such as [15]:

- $\quad$ Pro-active Routing

- $\quad$ Protocol Reactive

- $\quad$ Routing Protocol Hybrid

\subsection{Pro-active Routing Protocol}

The Pro-active routing protocol is also known as a tabledriven routing protocol, because in pro-active routing, a table is maintained to keep the route information. When a sender wants to send a file to a destination, this protocol finds an optimal path from the table [15] [16] [17]. Example of proactive routing protocols are:

- Destination Sequenced Distance Vector (DSDV)
- $\quad$ Optimized Link Source Routing (OLSR).

\subsection{Reactive Routing Protocols}

The reactive routing protocol is also known as an on-demand routing protocol. In a reactive routing protocol, when a sender wants to send a packet to a destination using an algorithm this protocol finds out the optimal path from source to destination [18].

Examples of reactive routing protocols are AODV (Ad hoc On-Demand Distance Vector) and DSR (Dynamic Source Routing).

\subsection{AODV routing protocol}

An example of an on-demand or reactive protocol is the AODV protocol, nodes neither contain any routing knowledge nor tend to periodic the exchanges of the routing table. AODV relies on a broadcast route discovery mechanism, which is used to dynamically maintain route table entries at interior nodes. However, AODV relies on dynamically establishing route table entries at internal nodes, instead of source routing. This distinction pays off in networks with numerous nodes, where a large scale of overhead is adequate holding source routes in each packet. To control the most current routing information between intermediate nodes, AODV takes the concept of destination sequence numbers from DSDV.

\section{METHODOLOGY}

The growing challenges of wireless networks can be effectively mitigated using SDN. The user's QoS is greatly improve because it provides a logically centralized control plane for the networks. The centralized feature of SDN makes a significant reduction in control traffic that increases rapidly with the introduction of new services. The SDN based wireless structure can operate the multiple controllers in a given area to allow users to connect to any Access Points that the operator may not belong to. In this paper work, a reactive routing protocol (AODV) is implemented in SDN.

\subsection{Working Procedure}

This experimentation demonstrates the implementation of AODV in SDN in a wireless network. SDN supports different QoS functionality and adaptability in a wireless network. It enhances radio access networks with centralized programmability [19]. Routing protocols is one of the best control plane components. In this method, AODV is used as a routing protocol to determine the best path from source to destination through a network.

How a packet is transformed or being dropped is the fundamental functionality of the data plane and the features of QoS have evaluated on this plane. Mininet-Wifi [20] network simulator is chosen for constructing the large-scale wireless network and analyzing the QoS performance. AODV establishes a route from the sender to its destination when a node wants to send information. AODV avoids routing loop using sequence number (SN) and this feature makes it different from other on-demand routing protocols. Route discovery and route maintenance are the operators which are treated as basic operators for AODV.

Figure 1 illustrates the interaction between two cars through a controller. A network area is constituted through an access point. If a destination car doesn't locate in any access point, it can't be reachable. For controlling the interaction between two cars and find out the optimal path between them, a central controller is used. 

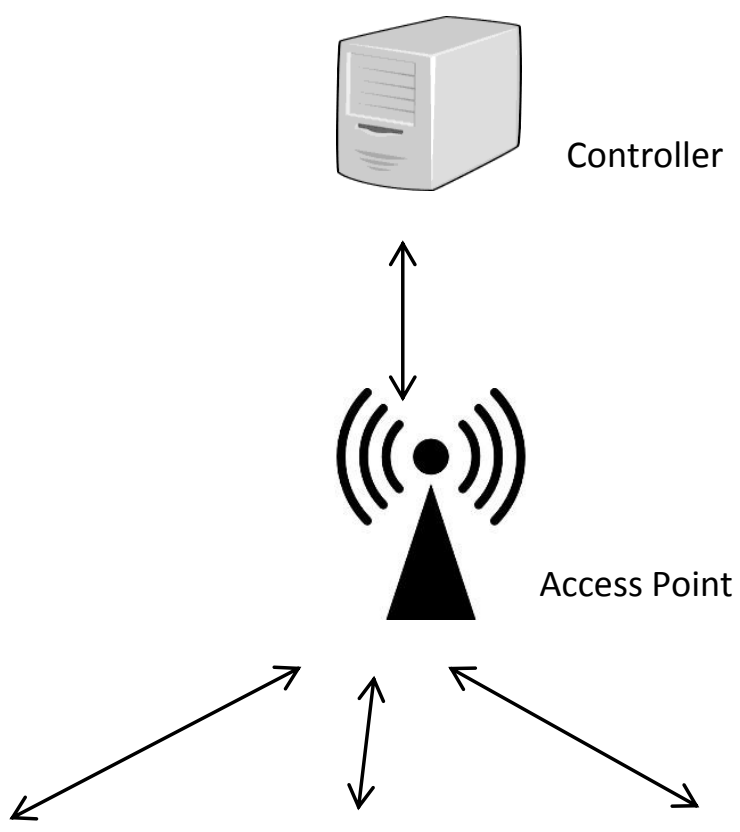

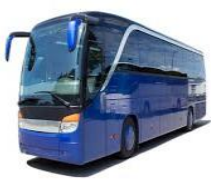

Node 1 or STA1

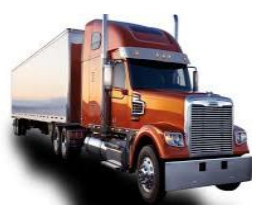

Node 2 or STA2

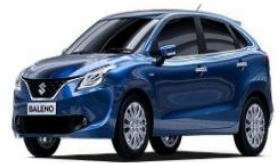

Node 3 or STA3

Figure 1: Communication between Source and Destination Using AP and Controller in SDN

\subsection{Performance Metrics}

Maximum packet delay and Average packet travel speed are used as a performance metrics to compare the result with a traditional network.

\section{- Maximum Packet Delay:}

The following formula is used to calculate Maximum Packet Delay [21]:

$$
\mathrm{d}=\max \left(\mathrm{t}_{\mathrm{r}}-\mathrm{t}_{\mathrm{t}}\right) \quad \text { for } \mathrm{i}=0 \text { to } \mathrm{n} \text {; }
$$

where,

$\mathrm{d}=$ the maximum delay,

$\mathrm{n}=$ the number of transmitted packet,

$\mathrm{t}_{\mathrm{r}}=$ time when the packet is received and

$\mathrm{t}_{\mathrm{t}}=$ the packet is transmitted.

\section{- Average Packet Travel Speed:}

The average packet travel speed is used as another metric in this work. The neighbor is essential for the dispersion of the position of a vehicle. The average travel speed of the packet observation will disclose us which routing protocol has done better jobs in disseminating the warning information. Using the following formula, Maximum Average Packet Travel Speed is calculated [21]:

$$
V=\frac{1}{n} \sum_{i=1}^{n} \frac{S}{\mathrm{t}-\mathrm{t}} \mathrm{t}
$$

Where,

' $n$ ' denotes the number of received packet, and 's' denotes the distance when the packet is transmitted.

\subsection{Network Design and Implementation}

Mininet-wifi is a simulator used to implement the AODV routing protocol in SDN. It is a SDN network emulator. Using Wireshark and Iperf simulation results are captured in this work. All the task is accomplished under the Linux platform and Operating System "Linux 14.04" is used to set up the environment. The other versions of Linux do not provide good support to run mininet projects. Mininet-wifi is used here as the simulator. The area is defined as $100 * 100 \mathrm{~m}$ with the simulation time 10 seconds. First, a network is designed for 3 cars then sequentially networks for 4, 5, 7, 9 cars are created and analyze the performance. Table 1 , shows the parameters and the corresponding specification for the environment setup.

Table 1: Simulation Environment Setup

\begin{tabular}{|l|l|}
\hline Parameters & Specification \\
\hline OS & Linux 14.04 \\
\hline Network simulator & Mininet-wifi \\
\hline Simulation time & 10 seconds \\
\hline Simulation area & $100 \mathrm{~m}^{*} 100 \mathrm{~m}$ \\
\hline Number of vehicles & $3-12$ cars \\
\hline Transmission range & $250 \mathrm{~m}$ \\
\hline MAC Protocol & IEEE $802.11 \mathrm{~b}$ \\
\hline Channel Type & Wireless \\
\hline
\end{tabular}




\section{RESULT AND ANALYSIS}

To compare the performances, maximum packet delay and average packet travel speed are considered as the comparison parameter. Mininet-wifi is used as the simulator to implement AODV in SDN. Simulation time is set 10 seconds in a predefined simulation area and routing protocol AODV. The network is analyzed for the number of $3,4,5,7,9$ and 11 nodes.

\section{Reachable and Unreachable Car}

Figure 1 depicts the communication between vehicles using a controller. Access points create a network area. A car is denoted as a node or station or STA. If a destination car locates inside the network then it is reachable. After doing the ping operation response message are created as shown in Figure 2. If a destination car doesn't locate at any access point, it can't be reachable. Unreliability of car shows in Figure 3.

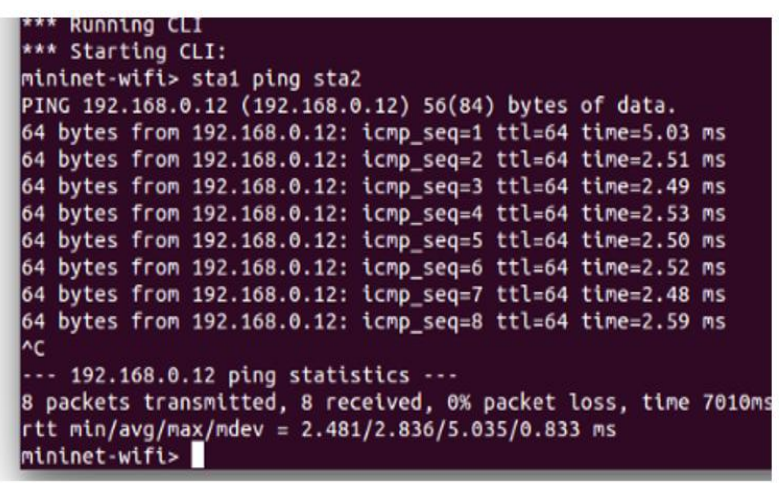

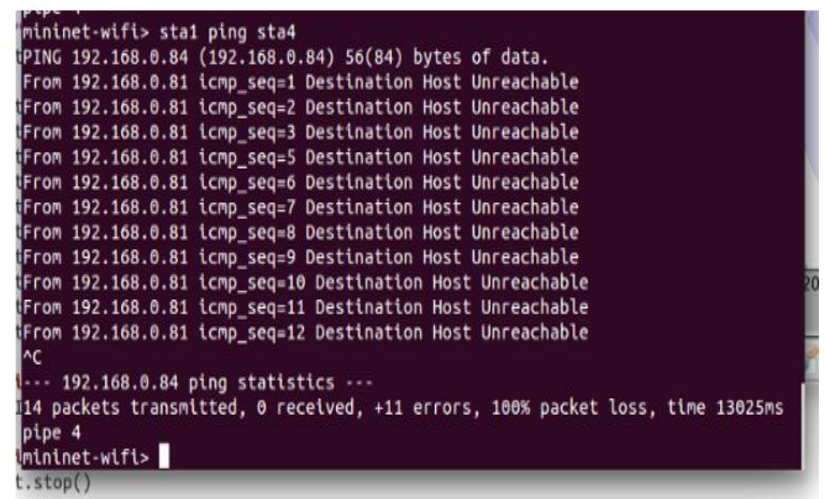

Figure 3: Unreachable car

\section{Maximum packet Delay}

The result is compared with traditional network architecture [21]. Comparison of Maximum Packet Delay between SDN and traditional network architecture for AODV is given in figure 4. From figure 4, it is observed that for the increasing number of nodes always SDN provides better performance than traditional networks.

Figure 2: Reachable car

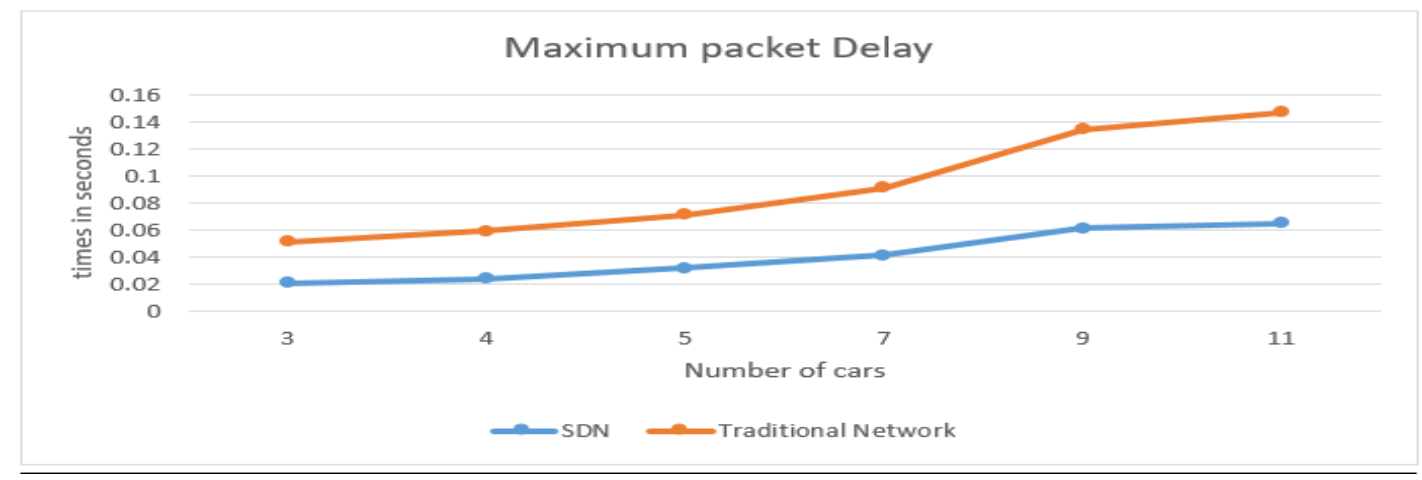

Figure 4: Maximum packet Delay for Software-defined Network Versus Traditional Network

\section{Average Time Speed of Packet}

The result is compared with [21] in figure 5. Here it is seen that the average time speed of packet is always maximum than a traditional network but with the increasing number of cars, average travel speed of packet is decreased not only in the traditional network but also in the SDN network. 


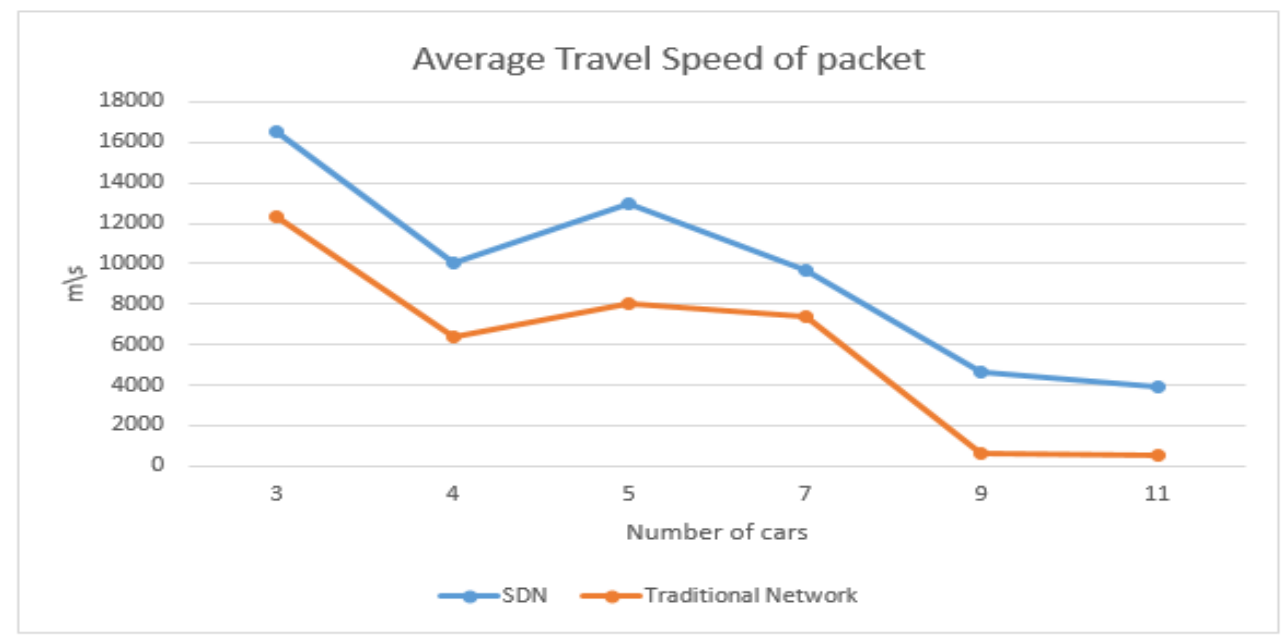

Figure 5: Average Time Speed of Packet for Software-Defined Network Versus Traditional Network

\section{CONCLUSION AND FUTURE WORKS}

Theoretically, it is known that SDN is dissimilar from other types of network architecture because the control plane is programmable, and the control and data planes are separated in SDN. The SDN architecture for AODV is implemented in this paper and observes that a car or a station cannot interact with another car if any of them is outside of any access point and two cars can interact if they are in any access point in the network.

After the comparison of the results, it is found that the maximum packet delay is less than other traditional network architecture for VANET in SDN. Besides, in terms of the time speed of the packet, SDN architecture is faster than the normal traditional networks.

There are some plans to inspect and examine the following related problems, extensions:

- It will be tried to analyze the security of VANET in SDN.

- It will be compared with throughput, jitter and other QoS metrics for this implemented architecture with other traditional architectures.

\section{REFERENCES}

[1] "Software-defined networking ( $\operatorname{sdn})$ definition https://www.opennetworking.org/sdnresources/sdndefinition."

[2] I. Z. Bholebawa, R. K. Jha, and U. D. Dalal, "Performance analysis of proposed openflow-based network architecture using mininet," Wireless Personal Communications, vol. 86, no. 2, pp. 943-958, 2016.

[3] B. A. A. Nunes, M. Mendonca, X.-N. Nguyen, K. Obraczka, and T. Turletti, "A survey of software-defined networking: Past, present, and future of programmable networks," IEEE Communications Surveys \& Tutorials, vol. 16, no. 3, pp. 1617-1634, 2014.

[4] M. Jerbi, S.-M. Senouci, R. Meraihi, and Y. GhamriDoudane, "An improved vehicular ad hoc routing protocol for city environments," in Communications, 2007. ICC'07. IEEE International Conference on. IEEE, 2007, pp. 3972-3979.
[5] Y. Liu, J. Bi, and J. Yang, "Research on vehicular ad hoc networks," in Control and Decision Conference, 2009. CCDC'09. Chinese. IEEE, 2009, pp. 4430- 4435.

[6] C. Perkins, E. Belding-Royer, and S. Das, "Ad hoc ondemand distance vector (aodv) routing," Tech. Rep., 2003.

[7] P. Lv, B. Zheng, and Z. Zhou, "Simulation of vanet in a more realistic scenario," in Wireless Communications, Networking and Mobile Computing (WiCOM), 2011 7th International Conference on. IEEE, 2011, pp. 1-3.

[8] X. Fang, K. Chai, Y. Alfadhl, and Y. Sun, "Evaluation of ad-hoc routing protocols in vehicular ad-hoc network using opnet," in ITS Telecommunications (ITST), 2011 11th International Conference on. IEEE, 2011, pp. 3944.

[9] R. S. Shukla and N. Tyagi, "Performance evaluation of mobility model and routing protocols for inter vehicular communication system," in Emerging Trends in Networks and Computer Communications (ETNCC), 2011 International Conference on. IEEE, 2011, pp. 263 266.

[10] V. Rajeshkumar and P. Sivakumar, "Comparative study of aodv, dsdv and dsr routing protocols in manet using network simulator-2," International Journal of Advanced Research in Computer and Communication Engineering, vol. 2, no. 12, pp. 2278-1021, 2013.

[11] R. K. Habib M, "A survey: Use of sdn on dsdv routing protocol in vanet," International Journal of Scientific Engineering and Science, vol. 1, no. 11, pp. 53-57, 2017.

[12] B. K, "Software-defined networking (sdn): a survey, security and communication networks," vol. 9, no. 18, pp. 5803-5833, 2016.

[13] "Floodlight controller, floodlight documentation, for developers, architecture. [online]. retrieved from: http://www.projectfloodlight.org/floodlight/."

[14] T. W. Chim, S.-M. Yiu, L. C. Hui, and V. O. Li, "Vspn: Vanet-based secure and privacy-preserving navigation," IEEE Transactions on Computers, vol. 63, no. 2, pp. 510-524, 2014. 
[15] M. Keshtgary and V. Babaiyan, "Performance evaluation of reactive, proactive and hybrid routing protocols in manet," International Journal on Computer Science and Engineering, vol. 4, no. 2, p. 248, 2012.

[16] X. Sun and X.-M. Li, "Study of the feasibility of vanet and its routing protocols," in Wireless Communications, Networking and Mobile Computing, 2008. WiCOM'08. 4th International Conference on. IEEE, 2008, pp. 1-4.

[17] F. Cuomo, S. D. Luna, U. Monaco, and T. Melodia, "Routing in zigbee: benefits from exploiting the ieee 802.15. 4 association tree," in Communications, 2007. ICC'07. IEEE International Conference on. IEEE, 2007, pp. 3271-3276.

[18] A. Dixit, F. Hao, S. Mukherjee, T. Lakshman, and R. R. Kompella, "Elasticon; an elastic distributed sdn controller," in Architectures for Networking and Communications Systems (ANCS), 2014 ACM/IEEE Symposium on. IEEE, 2014, pp. 17-27.

[19] C. J. Bernardos, A. De La Oliva, P. Serrano, A. Banchs, L. M. Contreras, H. Jin, and J. C. Z'u niga, "An architecture for software defined wireless networking," IEEE wireless communications, vol. 21, no. 3, pp. 52-61, 2014.

[20] https://sdncentral.ir/wpcontent/uploads/2017/07/mininetwifi-draftmanual.pdf [accessed date: 15/04/2020].

[21] G. Z. Santoso and M. Kang, "Performance analysis of aodv, dsdv and olsr in a vanets safety application scenario," in Advanced communication technology (ICACT), 2012 14th international conference on. IEEE, 2012, pp. 